\title{
Reflexiones acerca prevalencia de la hipertensión arterial esencial y los efectos del ejercicio físico con fines profilácticos y terapéuticos
}

\author{
Reflections on the prevalence of essential hypertension and the effects of \\ physical exercise for prophylactic and therapeutic purposes
}

\author{
Msc. Eugenio González Pérez ${ }^{1}$, Drc. Carlos Manuel Fuentes ${ }^{2} \&$ Drc. Luis Lázaro Arias \\ Rodríguez ${ }^{3}$
}

\begin{abstract}
.
"Currently, there is sufficient evidence that a regular exercise program reduces blood pressure by about $10 \mathrm{~mm} \mathrm{Hg}$. This is considered a significant reduction of blood pressure in the therapeutic area, greatly favored when compared to medical treatments because of its lack of side effects, in addition to the fact that it presupposes healthy lifestyle habits, increased quality of life and sustainable development, based on the low material and monetary cost required for its implementation and monitoring”.
\end{abstract}

\section{Resumen.}

"En la actualidad hay pruebas suficientes que un programa de ejercicios regulares reduce en unos $10 \mathrm{~mm} \mathrm{Hg}$. la tensión arterial, considerada como una reducción significativa de tensión arterial en el ámbito terapéutico, favorecida grandemente al compararlo con los tratamientos médicos por su carencia de efectos secundarios, además de que presuponen hábitos saludables de vida, aumento de la calidad de vida y un desarrollo sostenible, basado en el bajo costo

\footnotetext{
${ }^{1}$ Ciencias Médicas Mayabeque, Departamento Cultura Física jefe de Departamento, Mayabeque, Cuba, ergonzales@infomed.sld.cu iD https://orcid.org/0000-0002-7353-0182

2 Ciencias Médicas Mayabeque, Mayabeque, Cuba, cmfuentes@infomed.sld.cu 0002-7353-0183

${ }^{3}$ Ciencias Médicas Mayabeque, Mayabeque, Cuba, llarias@infomed.sld.cu ID https://orcid.org/0000-00027353-0184
} 
Keywords: tests, therapeutic, habits, lack, blood pressure. material y monetario que requieren para su puesta en práctica y seguimiento".

Palabras claves: pruebas, terapéutico, hábitos, carencia, tensión arterial.

\section{Introducción.}

El tratamiento de las enfermedades no transmisibles es unos de los problemas que más preocupan a las instituciones de salud y entre ellas, tiene un lugar cimero las enfermedades cardiovasculares, las cuales cobran miles de vidas anualmente, por lo que la estimación de los riesgos y las medidas que se pueden tomar son importantes en el control de estas. Mientras más tiempo permanezcan estas sin tratamiento alguno, mayor será el impacto sobre el organismo, que como agravante tiene que no surgen aisladamente, sino que constituyen "causa y efecto" entre sí, por lo que un individuo que padezca alguna, debe evaluarse con sumo cuidado para determinar la presencia de otros agentes agravantes de su salud.

\section{Desarrollo}

Dentro de los síntomas y enfermedades cardiovasculares, la hipertensión se ha denominado sabiamente el "asesino silencioso", porque no produce generalmente síntoma ninguno. Es importante, por lo tanto, para cualquier persona con factores de riesgo a padecer enfermedades cardiovasculares, hacerse chequear la tensión arterial con regularidad y proponerse la realización de cambios, adoptando estilos de vida apropiados. Tales recomendaciones son válidas para los individuos que tienen en general tensión arterial alta (inclusive la hipertensión que se consideran, clínicamente, como normal alta), antecedentes familiares de hipertensión, sufren de sobrepeso o son mayores de cuarenta años de edad. La hipertensión no tratada aumenta lentamente con el transcurso de los años, en casos raros (menos de un por ciento de los pacientes hipertensos), la tensión arterial asciende rápidamente, dando lugar a hipertensión maligna o acelerada. No obstante sea cual sea su carácter, esta es una enfermedad potencialmente mortal y debe tratarse de inmediato.

Dentro de los síntomas fundamentales que produce la hipertensión arterial se pueden contar: somnolencia, confusión, dolor de cabeza, náusea y visión borrosa y otros que se presentan con menos frecuencia. Recomendamos a los individuos hipertensos que deberán acudir al médico de inmediato si aparecen estos síntomas con frecuencia (5).

Para realizar la medición de la tensión arterial sistémica, que es la fuerza ejercida por la sangre contra las paredes de los vasos sanguíneos, se utiliza el estetoscopio y el esfigmomanómetro, la que a pesar de ser indirecta ofrece datos confiables del comportamiento de esta. La medición tensión arterial tiene dos componentes: 
- La tensión arterial sistólica, que mide la presión que ejerce la sangre sobre las arterias en fase de contracción del corazón (sístole) cuando es impulsada hacia los tejidos periféricos.

- La tensión arterial diastólica que mide la presión que ejerce la sangre sobre las paredes de las venas cuando el corazón se relaja (diástole) para permitir su entrada a la aurícula derecha (4).

La determinación de la tensión arterial en reposo es un componente básico de la evaluación previa del sujeto, tanto cuando asiste a consulta, como al someterse a los programas de rehabilitación, ya sea por medio del ejercicio u otros métodos. Las decisiones deben que fundarse en la media obtenida de dos o más mediciones de la tensión arterial. Esta se considera como riesgo cardiovascular cuando la tensión arterial sistólica es mayor a 140 mm Hg. y la tensión arterial diastólica es mayor a 90 mm Hg. A continuación, exponemos la clasificación del Comité conjunto de Detección, Evaluación y tratamiento de la tensión arterial para personas que no toman medicamentos antihipertensivos, no tienen enfermedades asociadas y son adultos mayores de 18 años (3).

Tabla 1. Clasificación del Comité conjunto de Detección, Evaluación y tratamiento de la tensión arterial para personas que no toman medicamentos antihipertensivos, no tienen enfermedades asociadas y son adultos mayores de 18 años.

TA Sistólica TA Diastólica Clasificación

\begin{tabular}{ccc}
\hline$<130$ & $<85$ & Normal \\
$130-139$ & $85-89$ & Normal alta \\
$140-159$ & $90-99$ & HTA leve (estadio 1) \\
$160-179$ & $100-109$ & HTA moderada (estadio 2) \\
$180-209$ & $110-119$ & HTA grave (estadio 3) \\
$>=210$ & $>=120$ & HTA muy grave (estadio 4)
\end{tabular}

Fuente: Elaboración propia.

Esta, según sus características y propósitos clínicos, se clasifica en dos categorías:

Hipertensión arterial primaria o esencial: tiene una prevalencia en más del $95 \%$ de los pacientes, ella puede ocasionarse por la existencia de múltiples mecanismos, como son trastornos del funcionamiento del sistema simpático, resistencia periférica de los vasos sanguíneos aumentada, ineficacia del control metabólico, alteraciones en la función baro- 
receptora de los vasos sanguíneos, etc., pero que en la mayoría de los casos no se puede definir o no existe una causa única conocida que produzca la hipertensión.

Hipertensión arterial secundaria: es causada por trastornos renales o endocrinos específicos, que aumentan la producción de hormonas como la adrenalina, noradrenalina, renina, cortisol, etc. Por su parte las enfermedades vasculares renales producen un aumento de la producción de renina, provocando la conversión de la angiotensina plasmática, que induce a una vasoconstricción periférica, y la liberación de aldosterona, que estimula la retención de sodio y agua y su disminución o escasez produce insuficiencia cardiaca. (3)

Sea cual sea la causa y categoría de la hipertensión, esta produce procesos patológicos importantes como son la hipertrofia del ventrículo izquierdo del corazón, al aumentar su tamaño (provocando insuficiencia cardiaca), arteriosclerosis, retinopatías y nefropatías. Estas enfermedades y lesiones suelen desarrollarse a lo largo de muchos años sin que se produzcan eventos mórbidos, lo que hace que a la hipertensión más peligrosa porque en la medida que se produzcan daños mayores, más difícil se hace combatir con efectividad los problemas de salud.

Además, se definen varios tipos de hipertensión arterial de acuerdo al comportamiento de la tensión sistólica y diastólica, como son (5):

Hipertensión arterial aislada: se produce cuando los valores de la tensión arterial se elevan mientras los de la tensión arterial diastólica se mantienen constantes, este tipo de hipertensión arterial se observa principalmente en personas en etapa de envejecimiento o adultos mayores, producto del aumento progresivo de la resistencia periférica de las paredes arteriales.

Hipertensión arterial diastólica: en este tipo se eleva a tensión arterial diastólica y la sistólica se mantiene normal.

Hipertensión arterial sístole-diastólica: en ella se elevan los valores tanto de la tensión arterial sistólica como de la diastólica.

Varios factores importantes pueden interactuar con influencias ambientales para producir presión arterial alta esencial, aunque ya planteamos anteriormente que no hay una causa conocida (4):

- Anomalías en el sistema angiotensina-renina: trastornos que afectan un grupo de hormonas conocidas como el sistema angiotensina-renina, el cual influye en todos los aspectos del control de presión arterial, entre otros la contracción de vasos sanguíneos, sal, el balance hídrico y el desarrollo de las células en el corazón. 
- Anomalías en el sistema nervioso simpático: algunas personas con hipertensión esencial pueden heredar las anormalidades del sistema nervioso simpático, que es la parte del sistema nervioso autónomo que controla el ritmo cardíaco, la presión arterial y el diámetro de los vasos sanguíneos.

- Resistencia a la insulina: las anormalidades metabólicas que causan resistencia a la insulina ocurren en la mitad de las personas con hipertensión. La resistencia a la insulina ocurre cuando los niveles de insulina son de normal a alto, pero el cuerpo no puede emplear la insulina para metabolizar el azúcar sanguíneo en las células musculares y almacenarla para lograr energía. La resistencia a la insulina es una característica principal en la diabetes tipo II o no dependiente a la insulina, la que a menudo va acompañada de presión arterial alta. Pero, no todas las personas con resistencia a la insulina tienen hipertensión, y no todas las que tienen presión arterial alta tienen este problema, lo que hace que, aunque exista algún enlace probable, la cadena de eventos que conducen a la presión arterial alta permanece especulativa. También el leptin o leptina, una sustancia relacionada tanto con la obesidad como con la resistencia a la insulina, también se encuentra elevada en muchas personas con presión arterial alta, aunque el papel juega en la presión arterial alta está bajo investigación.

- Niveles bajos de óxido nítrico: el óxido nítrico es uno de los factores más importantes que controlan la presión arterial. Si el endotelio no libera suficiente cantidad, los vasos sanguíneos se contraen, lo que incrementa la resistencia en la circulación periférica y como resultado, se eleva la presión arterial. Se ha comprobado que los pacientes que sufren hipertensión sistémica (presión arterial alta), hipertensión pulmonar (presión arterial alta en los pulmones) o alteraciones en la arteria coronaria, presentan alteraciones en el mecanismo de formación de este gas. (6)

- Niveles elevados del aminoácido homocisteína: los niveles sanguíneos anormalmente altos del aminoácido homocisteína están altamente vinculados con un mayor riesgo de coronariopatías y accidentes cerebrovasculares. Un estudio reciente ha indicado que los niveles altos de este aminoácido también pueden contribuir a la hipertensión sistólica causando que las arterias pierdan elasticidad y se tornen rígidas. Se dan niveles excesivos con carencias de las vitaminas B6, B12 y el ácido fólico. Parece que la homocisteína es tóxica para las células que revisten las arterias y que contribuye a que la sangre se coagule (4).

Se estima que millones de personas sufren de tensión arterial alta, cerca del $25 \%$ de todos los adultos y que dos tercios de la población mundial experimentará, por lo menos tensión arterial alta leve, antes de la edad de 65 años. Aunque la mayoría tienen hipertensión leve, inclusive esta enfermedad requiere de atención médica y una toma de conciencia en cuanto a cambiar el estilo de vida y la erradicación de los hábitos nocivos. Sólo el $20 \%$ de adultos tiene la tensión arterial bajo control, cerca de un tercio de los pacientes con tensión arterial alta sufren de sobrepeso (cualquier persona que sufre de sobrepeso corre el riesgo de padecer 
hipertensión, lo cual es un 50\% más que las personas con peso normal). En efecto, el aumento en la tensión arterial a medida que uno envejece puede deberse principalmente al aumento de peso. Por otra parte se ha comprobado por diferentes investigaciones que los niños y adolescentes que son obesos y los bebés con bajo peso al nacer tienen un mayor riesgo de padecer tensión arterial alta cuando alcanzan la edad adulta, cuyos datos los consideramos alarmantes teniendo en cuenta las creencias populares que ponen a la obesidad en los niños como muestra de buena salud y la incidencia de nacimientos con bajo peso, producidos generalmente por madres adolescentes o añosas, el hábito de fumar, la drogadicción y el alcoholismo en el embarazo, entre otros agravantes.

Algunos expertos creen que la hipertensión esencial puede heredarse en un 30\% a un 60\% de los casos, aunque varios genes, no sólo uno, probablemente están involucrados. Es muy difícil, sin embargo, diferenciar entre las influencias genéticas y ambientales, aún en los estudios con gemelos idénticos. Los investigadores recientemente han identificado un gen que puede indicar un riesgo mayor de accidente cerebrovascular en algunas personas que tienen tensión arterial alta, lo que puede ayudar a los médicos a determinar tratamientos específicos para estos pacientes.

Cerca del $40 \%$ de las personas con tensión arterial alta también tienen niveles de colesterol altos, aunque cualquier relación causal permanece poco clara aún. El estrés puede desempeñar un papel en esta asociación; en un estudio las personas con niveles de colesterol altos experimentaron un aumento precipitado de la tensión arterial al someterse a una prueba de estrés mental; los que tenían niveles de colesterol normales presentaron sólo un aumento moderado. Cuando el grupo de alto riesgo bajó su ingesta de colesterol, la tensión arterial descendió a niveles normales durante situaciones estresantes. Este resultado debería alentar a los médicos a concebir exámenes de riesgos de cardiopatías ante la presencia de personas con niveles de colesterol particularmente insalubres.

Varios estudios han vinculado la ocurrencia de hipertensión arterial con el estrés crónico, la depresión y la ansiedad, así como la manera de afrontamiento de la realidad con la presión arterial alta, tanto en los hombres como en las mujeres. Las personas que están ansiosas o deprimidas pueden tener dos veces el riesgo de tensión arterial alta que las que no tienen estos problemas. No está claro si estos trastornos del estado de ánimo contribuyen a la tensión arterial alta, debido a algún efecto fisiológico en los vasos sanguíneos o si pueden conducir a comportamientos como aumento de peso o abuso de alcohol, los cuales son también factores de riesgo de hipertensión.

Las personas que experimentan apnea del sueño, un trastorno en el cual la respiración se detiene breve reiteradamente durante el sueño, también tiene una incidencia mayor de hipertensión. Muchos expertos creen que existe una relación causal entre el trastorno del sueño y la presión arterial alta (4). Tenemos referencias de que la obesidad puede ser un 
factor coadyuvante a la ocurrencia de la apnea del sueño que mejora con la pérdida de peso. (6)

Los cambios en las estaciones pueden influir en las variaciones de la tensión arterial, haciendo que aumente durante los meses fríos y descienda durante el verano (este efecto estacional es particularmente alto entre los fumadores). Mientras el resfrío puede angostar los vasos sanguíneos, otro estudio reveló que la falta de luz estaba asociada con una tensión arterial más alta. Un estudio reciente informó que las personas con tensión arterial normal en descanso, cuya tensión aumenta a niveles anormalmente altos durante ejercicios de máquinas de rueda, pueden correr el riesgo de hipertensión esencial en el futuro. También los anticonceptivos orales, inclusive las dosis bajas, pueden aumentar el riesgo de presión arterial alta en las mujeres.

Dado que la hipertensión puede causar que ciertos órganos se deterioren con el transcurso del tiempo, los pacientes que no la controlan se enfrentan con una expectativa de vida menor. La tensión arterial alta contribuye en un $75 \%$ a todos los accidentes cerebrovasculares y ataques cardíacos.

Comparadas con los individuos normales, las personas hipertensas pueden correr un riesgo hasta diez veces mayor de accidente cerebrovascular y cinco veces mayor de un ataque cardíaco, según la gravedad de la hipertensión. El riesgo de desarrollar insuficiencia cardiaca congestiva es también significativamente más alto. Las personas cuya tensión arterial ha causado hipertrofia ventricular izquierda (engrosamiento de los músculos en el lado izquierdo del corazón que causa aumento de volumen) permanecen a riesgo de accidentes cerebrovasculares, ataques cardíacos, muerte repentina e insuficiencia cardiaca incluso después de que la tensión arterial está bajo control médico. En los estudios con animales, los investigadores han encontrado que cuando las células del corazón se agrandan como respuesta a la tensión arterial alta, experimentan cambios moleculares que causan una secreción anormal de calcio, un mineral crucial para la adecuada contracción del corazón. Este defecto parece ser irreversible, haciendo que la detección y la reducción de la hipertensión, lo antes posible, sea una medida preventiva sumamente importante. La tensión arterial alta causa un $30 \%$ de todos los casos de insuficiencia renal que requieren diálisis y operaciones de transplante (esta tasa ocupa el segundo lugar después de la diabetes).

Alguna forma de disfunción sexual ocurre en un 17\% de los hombres hipertensos, producida a menudo por los medicamentos que se prescriben, pero hay indicios de que el trastorno mismo puede deteriorar la función sexual, aunque la impotencia relacionada con la hipertensión es tratable. Las mujeres con tensión arterial alta, independientemente de los medicamentos, encontraban difícil el lograr satisfacción sexual y sufrían de lubricación vaginal impedida. Unido a esto debemos tener presente el alto nivel de ansiedad, depresión, baja autoestima y, el consiguiente estrés que se produce en estos casos. 
La tensión arterial alta crónica se asocia con el deterioro mental, en especial la memoria y atención a corto plazo. Un estudio ha encontrado que las personas de edad media con tensión arterial sistólica alta corren un riesgo mayor de función mental precaria en la en los años siguientes; mientras mayor sea la tensión arterial, mayor es el riesgo. Por otra parte la hipertensión arterial entre los hombres ancianos también se asocia con un riesgo mayor de demencia y enfermedad de Alzheimer.

\section{Efectos secundarios de los tratamientos de hipertensión}

Uno de los asuntos más difíciles que los pacientes hipertensos confrontan, en particular aquellos con hipertensión primaria o esencial, es que el tratamiento puede hacer que se sientan peor que con los síntomas de la enfermedad, la cual es casi siempre asintomática. Los pacientes se enfrentan con una perspectiva de toda una vida para tomar medicamentos con efectos secundarios desagradables, reducir su ingesta de sal y tener cuidado con su dieta. Muchos de los medicamentos que tratan la presión arterial alta, por ejemplo, deterioran la función sexual, aunque esto es reversible de inmediato, una vez que se suspende el medicamento. Sin embrago, a pesar de los efectos secundarios de los medicamentos, realizando un programa de ejercicios dosificados se pueden revertir, en gran medida, las molestias provocadas por estos. El ejercicio físico provoca cambios a nivel físico, funcional, psico-social que hacen que estas personas vean la vida desde puntos de vista diferentes, aumentando su calidad de vida y afrontamiento de la realidad. Por estudios serios realizados, se ha comprobado que el ejercicio físico produce estados de pleno bienestar sobre las personas que lo practican de forma sistemática y dosificada.

Si se sospecha la hipertensión, el médico deberá obtener la siguiente información:

1. Historia médica familiar y personal, especialmente la incidencia de la tensión arterial alta, accidente cerebro-vascular, problemas del corazón, enfermedad renal o diabetes, etc.

2. Factores de riesgo de cardiopatía y accidente cerebro-vascular, incluyendo consumo de tabaco, ingesta de sal, obesidad, inactividad física y niveles de colesterol insalubres.

3. Cualquier medicamento (tanto con prescripción y sin prescripción) que se esté tomando.

4. Una revisión de los síntomas que pueden indicar hipertensión secundaria, como dolor de cabeza, palpitaciones del corazón, sudoración excesiva, calambres musculares o debilidad o urinación excesiva.

5. Cualquier factor emocional o ambiental que pueda afectar la tensión arterial. 


\section{Influencia del ejercicio físico sobre la hipertensión arterial}

La respuesta al ejercicio físico de la tensión arterial se caracteriza por un aumento casi lineal de la tensión arterial sistólica, combinado con una ligera disminución de la tensión arterial diastólica y un aumento directamente proporcional con respecto a los valores en reposo. Estas respuestas pueden proporcionar criterios válidos para el diagnóstico y tratamiento de la hipertensión arterial.

La prescripción del ejercicio es útil en el tratamiento de la hipertensión arterial leve y moderada, particularmente en pacientes jóvenes y de mediana edad, debido a que se han detectado reducciones de hasta 8 a $10 \mathrm{~mm} \mathrm{Hg}$. en la tensión arterial sistólica y de hasta 5 a 8 mm Hg. en la diastólica. Hay ciertas evidencias de que un aumento elevado de la tensión arterial durante el ejercicio es una advertencia de un mayor riesgo a desarrollar hipertensión arterial, en cuanto a esto se han establecido las siguientes cifras de respuesta hipertensiva al ejercicio:

Respuesta Hipertensiva al esfuerzo:_es la respuesta donde la tensión arterial alcanza valores demasiado elevados, que se clasifican en:

Tabla 2. Respuesta de la tensión arterial.

$\underline{\text { T A Sistólica }}$

\begin{tabular}{cc}
\hline $190-210---$ Ligera & $110-120---$ Ligera \\
$220-240---$ Moderada & $120-130--$ Moderada \\
$+250---$ Severa & $+130--$ Severa \\
\hline
\end{tabular}

Fuente: Elaboración propia.

La mayor parte de los programas de ejercicios diseñados y utilizados implican una intensidad moderada en los ejercicios, empleando el trote, la caminata o la bicicleta. Parece ser que la intensidad alta no ofrece ventajas en la reducción de la tensión arterial, aunque algunos estudios sugieren el entrenamiento con pesas como método seguro y ventajoso para pacientes que padecen de hipertensión leve y moderada, pues mejoran la fuerza y la potencia muscular de los sujetos y los prepara de forma integral para su inclusión dentro de la sociedad (3). Actualmente en nuestro país se ha ido ganando conocimiento y se ha ido poniendo en práctica estos métodos con pesas, utilizando pesos pequeños, por debajo del $30 \%$ de la fuerza máxima, con resultados muy alentadores.

La capacidad aeróbica se define como la capacidad para realizar un ejercicio dinámico que involucre los principales grupos musculares, de intensidad moderada o alta durante 
prolongados períodos de tiempo. La realización de este ejercicio depende del estado funcional de los sistemas respiratorio, cardiovascular y locomotor.

\section{Conclusiones}

Se considera que la capacidad aeróbica está relacionada con la salud porque:

- Un nivel bajo de capacidad respiratoria esta relacionado estrechamente con el riesgo de muerte prematura por diferentes causas y, específicamente, por enfermedades cardiovasculares.

- Un nivel alto de capacidad aeróbica está vinculado con la actividad física regular, lo que además la asocia con muchos beneficios para la salud, como son el aumento del consumo máximo de oxígeno, el gasto cardiaco, disminución de la frecuencia cardiaca en reposo, aumento del volumen sistólico y minuto, la capacidad de trabajo, entre otros parámetros.

\section{Referencias bibliográficas}

Rubalcaba Ordaz Luis, Canetti Fernández Santos. Salud vs. Sedentarismo. Editorial Pueblo y Educación, La Habana, 1989, página: 1.

Popov S.N. La Cultura Física Terapéutica. Editorial Pueblo y Educación, La Habana, 1988, página: 141.

American College of Sorts Medicine. Manual de Consulta para el Control y Prescripción del Ejercicio, Editorial Paidotribo, Barcelona, España, 2000, página:171.

Freggario Eduardo Luis. Presión arterial, artículo científico. National Heart, Lung and Blood Institute, http://www.nhlbi.nih.gov/nhlbi/nhlbi.htm

Manidi Marie-Jose, Michel Jean-Pierre. Actividad Física para adultos con más de 55 años. Cuadros clínicos y programas de ejercicios. Sao Paulo, Brasil. Editorial Manole Ltda. , 2001, página:11.

Enciclopedia Encarta 2001. Apnea.

Colectivo de autores. Folleto de Preparación Física, apuntes para la asignatura. EIEFD, La Habana, 2001. página:

García Manso J. Manuel, Valdivieso Navarro M., Ruíz Caballero J. Antonio. Pruebas para la Valoración de la Capacidad Motriz en el Deporte, evaluación de la condición física. Editorial Gimnos, Barcelona, 2001. (código: 135)

iam E. Pretice. Técnicas de Rehabilitación en la Medicina Deportiva, 2da edición. Editorial Paidotribo, 2000 
American College of Sport Medicine. Manual para la valoración y prescripción del ejercicio. Editorial Paidotribo, Barcelona, 2001.

"Tensión arterial." Enciclopedia® Microsoft® Encarta 2001. @ 1993-2000 Microsoft Corporation.

Artículos de Revisión. Dra Cabrera Rojo. Iliana. Fisiopatología de la hipertensión arterial esencial. Hospital Clínico Quirúrgico Docente "General Calixto García" 


\section{PARA CITAR EL ARTÍCULO INDEXADO.}

González Pérez, E., Fuentes, C. M., \& Arias Rodríguez, L. L. (2018). Reflexiones acerca prevalencia de la hipertensión arterial esencial y los efectos del ejercicio físico con fines profilácticos y terapéuticos. Anatomía Digital, 1(1), 16-27. https://doi.org/10.33262/anatomiadigital.v1i1.1038

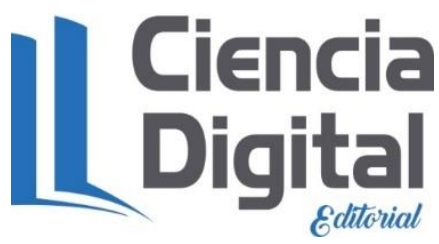

El artículo que se publica es de exclusiva responsabilidad de los autores y no necesariamente reflejan el pensamiento de la Revista Anatomía Digital.

El artículo queda en propiedad de la revista y, por tanto, su publicación parcial y/o total en otro medio tiene que ser autorizado por el director de la Revista Anatomía Digital.
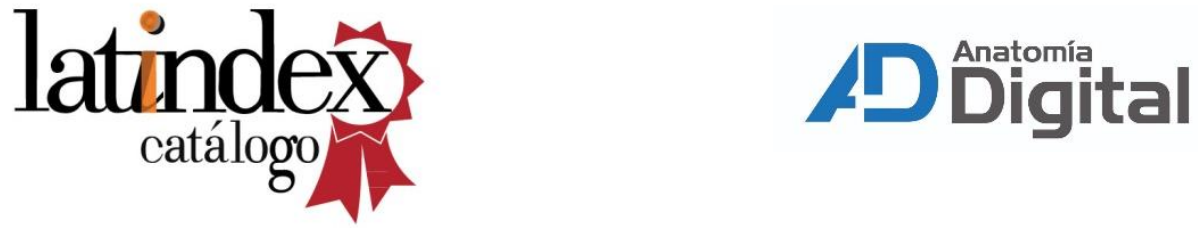